\title{
Orbital granulocytic sarcoma
}

Frank A Stockl, Angela M Dolmetsch, M Antonia Saornil, Ramon L Font, Miguel N Burnier Jr

\begin{abstract}
Aim-Orbital granulocytic sarcoma is a localised tumour composed of cells of myeloid origin. Histological diagnosis can be difficult in patients with poorly differentiated orbital tumours and no evidence of systemic leukaemia. The naphthol AS-D chloracetate esterase (Leder stain) and immunohistochemical stains for lysozyme and MAC387 were used to determine the staining characteristics of these tumours. A case series of seven patients with orbital granulocytic sarcoma is presented.
\end{abstract}

Methods-Seven patients with orbital granulocytic sarcoma were studied. Haematoxylin and eosin, Leder, and lysozyme stained sections were available in seven cases. Unstained formalin fixed paraffin embedded sections of seven cases were available for immunohistochemical evaluation using the avidin-biotin-complex technique for MAC387.

Results-The mean age of presentation of the orbital tumour was 8.8 years. Four patients presented with an orbital tumour before any systemic manifestations of leukaemia. In two cases the diagnosis of the orbital tumour and systemic leukaemia was made simultaneously. There was one case of established systemic myeloid leukaemia in remission with the subsequent development of orbital granulocytic sarcoma. Six of seven cases $(86 \%)$ were positive for the Leder stain. Five of seven cases (71\%) showed positive immunoreactivity with lysozyme. The immunohistochemical stain for MAC387 was positive in all seven cases $(100 \%)$ including one case that was negative for both lysozyme and Leder stains.

Conclusions-Orbital granulocytic sarcoma is a tumour that affects children and can present with rapidly progressive proptosis. This tumour may develop before, during, or after the occurrence of systemic leukaemia. The combination of Leder and lysozyme stains is useful in the diagnosis of orbital granulocytic sarcoma. MAC387 may be a more reliable marker for orbital granulocytic sarcoma.

(Br F Ophthalmol 1997;81:1084-1088)

Granulocytic sarcoma is a localised tumour comprised of malignant cells of myeloid origin. The first published report of granulocytic sarcoma was in 1811 by Allen Burns who described a green tumour involving the orbit. ${ }^{1}$
Because these tumours can exhibit a characteristic green colour they were named chloroma. The green colour is caused by exposure of the enzyme myeloperoxidase to ultraviolet light. However, the gross appearance of these tumours is variable and up to $30 \%$ of granulocytic sarcomas do not display a green colour. This led Rappaport to rename these tumours granulocytic sarcoma in $1966 .{ }^{2}$ Granulocytic sarcoma has been known by a variety of names including myeloblastoma, myelocytoma, chloroleukaemia and the World Health Organisation currently favours the name myeloid sarcoma. $^{3}$

Granulocytic sarcoma may occur as a manifestation of a well established systemic myelogenic leukaemia or it may precede systemic manifestations of peripheral blood and bone marrow. This tumour may present in association with different types of myeloid leukaemia including acute myelogenous leukaemia, chronic myelogenous leukaemia with or without blast crisis, and other myeloproliferative disorders. ${ }^{4}$ When granulocytic sarcoma precedes the development of leukaemia it usually heralds the development of peripheral blood and bone marrow involvement within 1 year. ${ }^{45}$

Granulocytic sarcoma may be found in a variety of locations throughout the body including the orbit. ${ }^{467}$ Orbital granulocytic sarcoma typically affects children and young adults and must be differentiated from other orbital tumours that typically affect this age group. ${ }^{6}$ When a patient has a history of leukaemia and then develops orbital granulocytic sarcoma the diagnosis is usually not difficult. However, when the orbital tumour precedes the development of systemic leukaemia the diagnosis can be difficult for both clinician and pathologist. In addition, these tumours are often poorly differentiated making diagnosis more difficult. ${ }^{8}$ The differential diagnosis includes malignant lymphoma, rhabdomyosarcoma, and neuroblastoma.

Histological features of myeloid differentiation such as eosinophilic granules within the cytoplasm may be helpful in establishing a diagnosis. However, these findings are often absent and not specific to granulocytic sarcoma. ${ }^{9}$ The naphthol-AS-D chloracetate esterase stain (Leder stain) greatly improved the ability of the pathologist to identify cells that contain myeloperoxidase. Similarly, immunohistochemical staining using antilysozyme antibodies for detection of lysozyme has proved useful in the identification of myeloid cells. Each of these stains in combination or alone is helpful when positive; however, 
Table 1 Clinical features of cases of orbital granulocytic sarcoma (OGS)

\begin{tabular}{llllll}
\hline Case & Age & Sex & Proptosis & Presentationt & Interval $\neq$ \\
\hline 1 & 9 years & Male & R & OGS & 11 months \\
2 & 9 years & Female & L & OGS & 25 days \\
3 & 11 years & Female & R & OGS & 3 months \\
4 & 10 years & Male & L & OGS & 3 months \\
5 & 5 months & Male & L & OGS and leukaemia & Simultaneous \\
6 & 8 years & Male & R & OGS and leukaemia & Simultaneous \\
7 & 14 years & Male & R & Leukaemia & 8 months' remission
\end{tabular}

^All seven patients presented with rapidly progressive proptosis.

fOGS denotes patients who presented with orbital granulocytic sarcoma before any evidence of systemic leukaemia. OGS and leukaemia denotes patients who developed orbital granulocytic sarcoma and leukaemia simultaneously. Leukaemia denotes patients who had leukaemia before developing orbital granulocytic sarcoma.

$\ddagger$ Time interval between orbital granulocytic sarcoma and systemic leukaemia

a negative stain does not rule out granulocytic sarcoma. ${ }^{9}$

We present seven well documented cases of orbital granulocytic sarcoma. We studied the clinical features and compared the use of the Leder stain and two immunohistochemical markers for myeloid cells-lysozyme and MAC $387 .^{10}$

\section{Materials and methods}

Seven cases of well documented orbital granulocytic sarcoma were studied at the McGill Ophthalmic Pathology Laboratory. Three of the cases were kindly provided by Dr R Font from the Baylor College of Medicine. The other four cases were obtained from the McGill Ophthalmic Pathology Registry. Cases were included in the study only if the myeloid origin of the tumour could be established by either positive Leder or lysozyme staining or the documentation of acute myeloid leukaemia in the bone marrow or peripheral blood. Clinical information was assessed for all seven cases. Histological features were evaluated using haematoxylin and eosin stained sections for degree of differentiation, mitotic figures, malignant features, and the presence of cytoplasmic eosinophilic granules. Paraffin blocks were obtained in all seven cases. The Leder stain was available for all seven cases. The Leder stain was considered positive if more than $5 \%$ of the cells stained positive. Imunohistochemical stains for lysozyme, MAC387, LCA, CD20 (L26), CD 43 (MT-1), CD 45 (LCA), desmin, and cytokeratin (CAM 5.2) were performed using the standard avidin-biotin-complex technique previously described. ${ }^{11}$ Appropriate positive and negative controls were used for all immunohistochemical stains. Stains were considered positive if staining could be unequivocally demonstrated in tumour cells. The anti-lysozyme, MAC387, CD 20, CD 45, and desmin antibodies were obtained from Dako Corporation, Carpinteria, CA, USA. Cytokeratin antibodies were obtained from Becton Dickinson Immunocytotometry Division, San Jose, CA, USA. CD 43 antibodies were obtained from Biotest Diagnostics Denville, NJ, USA. Five cases of orbital lymphoma were obtained and stained with lysozyme and MAC387 as negative controls.

\section{Results}

Clinical data are summarised in Table 1. The age of presentation ranged from 5 months to
14 years with a mean age of 8.8 years. The orbital tumour preceded the development of systemic leukaemia in four cases. The mean time to development of systemic leukaemia was 5 months in these patients. In two cases the diagnosis of the orbital tumour and systemic leukaemia was made simultaneously. There was one case of established leukaemia in remission with development of orbital granulocytic sarcoma 8 months later.

\section{Selected case reports}

ORBITAL TUMOUR PRECEDES THE DEVELOPMENT OF LEUKAEMIA

Patient 1

A nine year old Latin-American boy was admitted to the hospital for rapidly progressive proptosis of the right globe. Two weeks before admission his right eye became painful. The patient had no previous illnesses.

Ophthalmic examination revealed right upper and lower lid oedema with proptosis and chemosis of the right eye (Fig 1A).

Computed tomography (CT) revealed a large irregular mass in the right superior orbit approximately $2 \mathrm{~cm}$ in diameter. Systemic examination, including a complete blood count and chest $x$ ray, was negative.

An excisional biopsy revealed a greenish tumour, $20 \mathrm{~mm}$ in diameter. Histopathological examination revealed an infiltrating neoplasm composed of poorly differentiated cells with round or reniform nuclei, prominent nucleoli, and many mitotic figures. The scant to moderate cytoplasm contained eosinophilic granules. The Leder, lysozyme, and MAC387 stains were positive (Fig 1B, C, D). A diagnosis of orbital granulocytic sarcoma was made.

Eleven months after the onset of the orbital tumour, the patient developed acute myelogenous leukaemia with blast cells in the peripheral blood. The patient underwent polychemotherapy.

\section{ORBITAL TUMOUR AND LEUKAEMIA DISCOVERED} SIMULTANEOUSLY

Patient 5

A 5 month old Spanish boy was admitted to the paediatric unit for evaluation of rapidly progressive proptosis of the left globe. Five days before admission his left eye became proptotic with left upper lid fullness and epiphora.

The initial ocular examination revealed unilateral left globe proptosis with downward and outward displacement of the eye without any signs of inflammation. A firm mass measuring approximately $5 \times 5 \mathrm{~mm}$ could be palpated in the superior nasal quadrant of the left orbit.

A complete physical examination was unremarkable. CT revealed a homogeneous mass with uniform contrast enhancement located in the left superior orbit, involving the lacrimal gland.

The Leder stain was positive. Immunohistochemistry was negative for actin-desmin and L26 (CD 20), and positive for LCA (CD 45), lysozyme, and MAC387.

A bone marrow biopsy demonstrated a monomorphic hypercellular proliferation of 

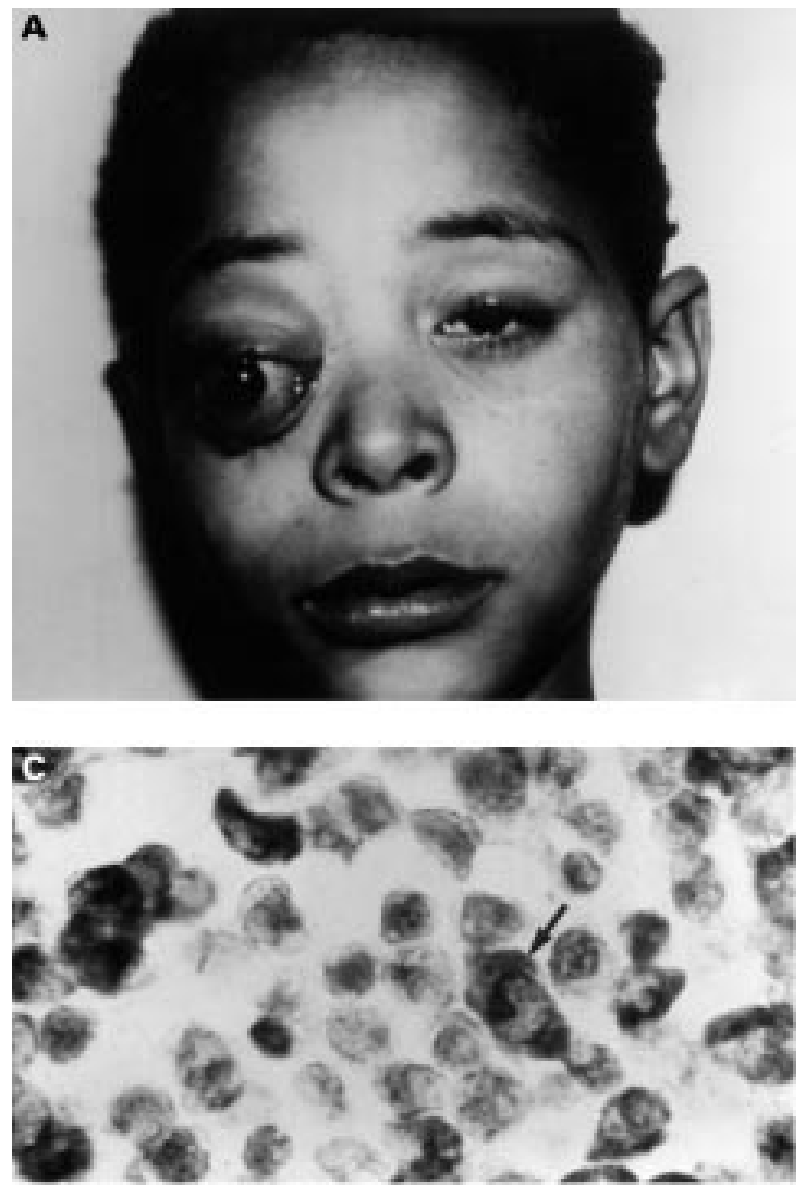

Figure 1 (A) Nine year old boy with proptotic right globe. (B)

Histopathological section of orbital tumour showing malignant proliferation invading fat (haematoxylin and eosin, $\times 26$ ). (C) High magnification showing immunoreactive cells (arrow) using lysozyme antibody (anti-lysozyme, $\times 128$ ). (D) Positive immunohistochemical stain (arrow) of the orbital tumour using MAC387 (MAC387 stain, $\times 104$ ).
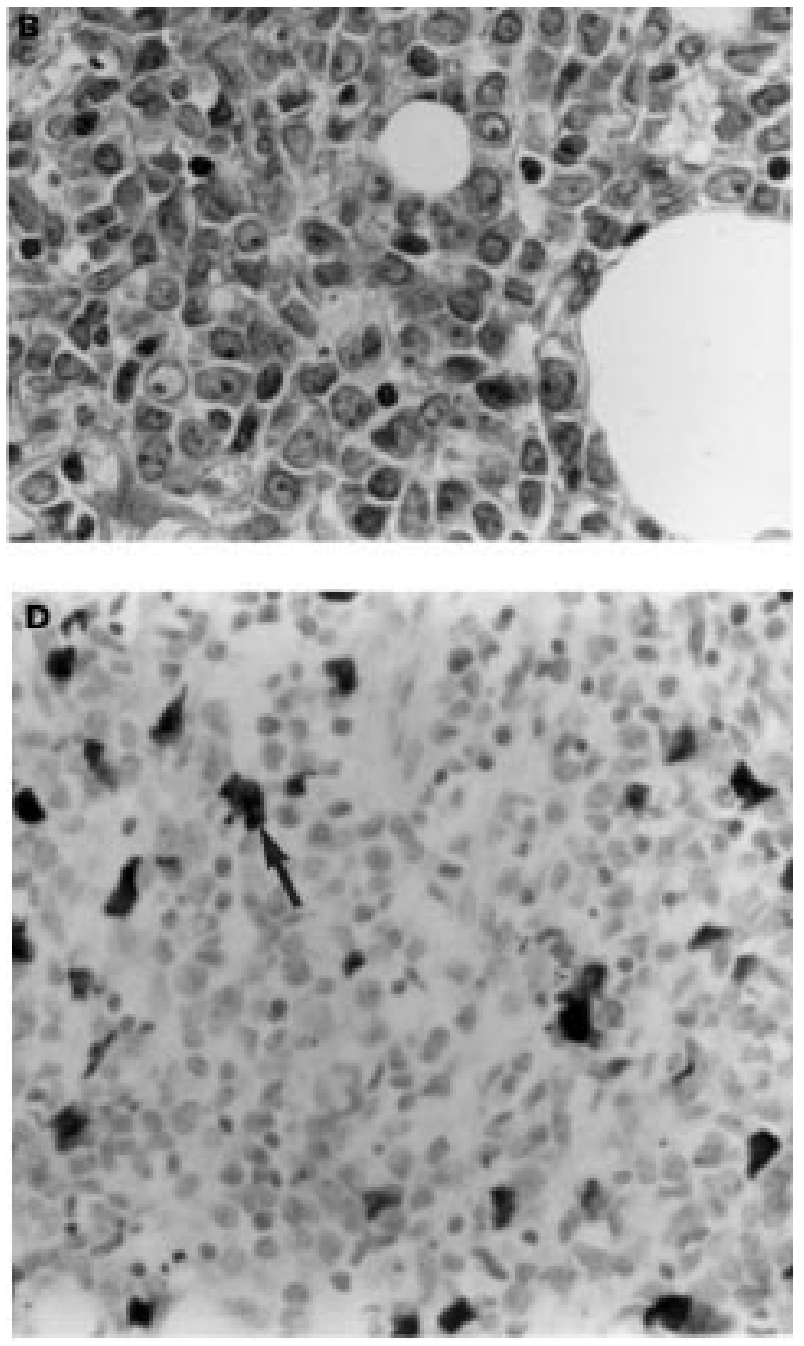

indented. There were prominent nucleoli and moderate mitotic activity. There is scant cytoplasm with no eosinophilic granules. The Leder stain was positive. A diagnosis of orbital granulocytic sarcoma was made. The patient was again treated with chemotherapy and has since been in remission.

\section{PATHOLOGY}

The seven tumours were classified as differentiated or poorly differentiated based on the presence or absence of granulocytic differentiation such as cytoplasmic eosinophilic granules and nuclear changes. Two of the cases were classified as differentiated and five cases were poorly differentiated. The Leder stain was positive in six of seven cases $(86 \%)$. Five of seven cases were positive for lysozyme (71\%), while all seven cases were positive for MAC387 $(100 \%)$ (Table 2). All seven cases were negative for CD 20, CD 43, desmin, and cytokeratin stains while only one cases was weakly positive for LCA. All five cases of orbital lymphoma demonstrated negative immunoreactivity with lysozyme and MAC387 (Table 2).

\section{Discussion}

Granulocytic sarcoma of the orbit can present in infancy to 61 years of age. ${ }^{6}$ Despite this broad age range of presentation, these tumours nous proliferation of poorly differentiated cells. The nuclei were round to oval with nuclei 
Table 2 Histopathological results of orbital granulocytic sarcoma cases

\begin{tabular}{lllll}
\hline Case & Gran diff $^{*}$ & Leder stain & Lysozyme stain & MAC387 stain \\
\hline 1 & Yes & Pos & Pos & Pos \\
2 & No & Pos & Neg & Pos \\
$3 \dagger$ & No & Neg & Neg & Pos \\
4 & No & Pos & Pos & Pos \\
5 & No & Pos & Pos & Pos \\
6 & No & Pos & Pos & Pos \\
7 & Yes & Pos & $5 / 7(71 \%)$ & $7 / 7(100 \%)$ \\
Total & $2 / 7(29 \%)$ & $6 / 7(86 \%)$ & \\
\hline
\end{tabular}

${ }^{\star}$ Granulocytic differentiation is based on the presence or absence of cytoplasmic eosinophilic granules in haematoxylin and eosin stained preparations.

tCase 3 initially had no marker of granulocytic sarcoma but 3 months after initial presentation developed systemic manifestations of AML.

typically present in children with a mean age of approximately 7 years. $^{6}{ }^{12}$ This is consistent with the mean age of 8.8 years in this study.

Granulocytic sarcoma can occur in a variety of locations throughout the body including the orbit. $^{46712}$ The most common sites of involvement are bone, soft tissues, lymph nodes, skin, and kidney. ${ }^{4}$ Bone involvement is usually subperiosteal and most frequently located in the vertebrae, sternum, orbit, and cranium. ${ }^{7}$ In Liu et al's study of 23 necropsy cases of granulocytic sarcoma, exophthalmos was present in seven cases and was the most common observable sign of tumour nodule. ${ }^{7}$

Certain paediatric populations are known have a higher incidence of orbital granulocytic sarcoma. Reports in the literature indicate that African, Middle Eastern, East Asian, and Latin American children have a disproportionately higher incidence. ${ }^{6}{ }^{72-14}$ This series also supports geographic variation of this condition as all of the cases were of Spanish descent. This study also revealed a slight male predominance which is in accordance with previously published literature. ${ }^{4} 67$

The clinical features of orbital granulocytic sarcoma can vary considerably. Proptosis is the most common presenting clinical sign. ${ }^{6712}$ In fact, Zimmerman and Font found 29 of 33 patients with orbital granulocytic sarcoma presented with signs of proptosis. ${ }^{6}$ Grossniklaus and Wojno described a case presenting as periorbital cellulitis which represented myeloblastic invasion of eyelid dermis. ${ }^{15}$ Various other presentations have been described including lacrimal gland involvement, eyelid tumour, post-traumatic, iris tumour, uveitis, conjunctival mass, and scleral mass. ${ }^{615-19}$

The most puzzling feature of orbital granulocytic sarcoma is its variable temporal onset relative to the development of systemic leukaemia. The occurrence of the orbital granulocytic sarcoma before the development of systemic leukaemia is not uncommon and was demonstrated in 29 of 33 cases ( $88 \%$ ) in the largest study of orbital granulocytic sarcoma by Zimmerman and Font. $^{6}$ Numerous other instances of this phenomenon can be found in the literature. ${ }^{18} 20$ The subsequent development of leukaemia usually occurs within 1 year. ${ }^{4-7}$ The AFIP series by Zimmerman and Font showed that the vast majority of cases had haematological evidence within 2 months. ${ }^{6}$ However, there are reports of prolonged intervals between the development of granulocytic sarcoma and systemic leukaemia varying from
2 to 16 years. ${ }^{521}$ In our series, the development of orbital granulocytic sarcoma occurred before any evidence of systemic leukaemia in four of seven cases (57\%) with a mean time to development of leukaemia in either the peripheral blood or bone marrow of 5 months.

The differential diagnosis of orbital granulocytic sarcoma can be challenging, particularly when there is no evidence of systemic leukaemia. It is most frequently confused with malignant lymphoma, but can also be confused with rhabdomyosarcoma, neuroblastoma, and other poorly differentiated tumours of childhood. ${ }^{6822}$ cannot be overemphasised that any tumour of the head and neck region, including the orbit, can potentially represent infiltration by malignant myeloid cells. ${ }^{23}$

In patients with known systemic leukaemia CT may help to distinguish between granulocytic sarcomas from haematomas and abscesses, which are possible complications of leukaemia. ${ }^{24}$ However, in patients who develop granulocytic sarcoma before leukaemia, CT or magnetic resonance imaging features are not specific enough to distinguish granulocytic neoplasms from other tumours. ${ }^{24} 25$

To establish the diagnosis of orbital granulocytic sarcoma a biopsy is often required. There is a correlation between the degree of differentiation of these tumours and the accuracy of the initial diagnosis. Blastic tumours are correctly diagnosed $38 \%$ of the time, poorly differentiated tumours $47 \%$ of the time, and well differentiated tumours $54 \%$ of the time. ${ }^{4}$ Typically, these tumours are composed of a monotonous invasive proliferation of malignant cells. The nuclei can vary from round in undifferentiated cells, to indented in more differentiated cells. Nucleoli are frequently prominent and the number of mitotic figures is variable. Histological differentiation between granulocytic sarcoma and lymphoma may be facilitated if the tumour reveals cells with large nuclei with blastic chromatin and prominent nucleoli as this finding is rare in lymphomas. ${ }^{9}$ The amount of cytoplasm and the presence of eosinophilic granules varies depending on the degree of differentiation of the cells. The cytoplasm is scant with no granules in undifferentiated cells whereas in more differentiated cells the cytoplasm may be more abundant and exhibit cytoplasmic granules. Granulocytic differentiation when present is clearly helpful in establishing the diagnosis. However, Niemen et al found in their series that only $20 \%$ of theses tumours have a significant degree of differentiation. ${ }^{4}$

Electron microscopy has been shown to be useful in establishing the diagnosis of granulocytic sarcoma. ${ }^{9}$ However, it is rarely used and since the development of the Leder stain and immunohistochemistry, electron microscopy is not required to establish the diagnosis. ${ }^{4}$

The development of the Leder and immunohistochemical stains have enhanced the ability of the pathologist to establish the diagnosis of granulocytic sarcoma. The Leder stain identifies cells with esterase activity. This stain can be used on formalin fixed paraffin embedded sections. ${ }^{9}$ The Leder stain has been shown to 
be positive in approximately $75 \%$ of cases in two retrospective series on granulocytic sarcoma. ${ }^{46}$ The results using the Leder stain in our study are similar to those previously published. A positive Leder stain is supportive of the diagnosis of granulocytic sarcoma but a negative stain does not rule out the diagnosis.

The immunohistochemical stain using antilysozyme antibody identifies cells that contain the enzyme lysozyme. The use of this marker in identifying tumours of myeloid origin has been established. ${ }^{46}$ The lysozyme stain has been shown to be positive in $60 \%$ to $89 \%$ of cases in previous studies of granulocytic sarcoma. ${ }^{46}$ More importantly, Neimen et al have shown that $98 \%$ of cases were identified positive for either Leder or lysozyme stains. ${ }^{4}$ Because one of the cases in our series gave negative results with both the Leder and lysozyme stains, we examined the use of monoclonal antibody MAC387. All seven cases stained with MAC387 demonstrated immunoreactivity. For the vast majority of cases, the combination of Leder and lysozyme stains will establish the diagnosis of granulocytic sarcoma. ${ }^{4}$ However, in cases of extremely poorly differentiated tumour in which the Leder and lysozyme stains give negative results, but clinical suspicion is high, MAC387 may prove useful in establishing the diagnosis.

\section{Conclusions}

Orbital granulocytic sarcoma may develop before, during, or after the occurrence of leukaemia in the peripheral blood and bone marrow. Histological diagnosis of granulocytic sarcoma can be difficult in children with no evidence of leukaemia, so a high index of suspicion by both the clinician and the pathologist is important in order to make the diagnosis. Special stains and immunohistochemistry play an important role in the diagnosis. The use of lysozyme and Leder stains in combination is useful in establishing the diagnosis of granulocytic sarcoma. MAC387 stain may provide additional information in cases in which the Leder and lysozyme stains give negative results and clinical suspicion is high.

Presented at The Association for Research in Vision and Ophthalmology (ARVO) Annual Meeting 1995 in Fort Lauderdale, Florida, USA

1 Burns A. Observation on the surgical anatomy of the head and neck. 4th ed. Baltimore, USA: Granville Sharp Pattison, 1823.
2 Rappaport H. Tumours of the hematopoietic system. In: $\mathrm{Sec}$ III, Fascicle 8, Atlas of tumour pathology. Washington DC: Armed Forces Institute of Pathology, 1966:241-63.

3 Zimmerman LE, Sobin LH. Histological typing of tumours of the eye and its adnexa. Geneva: World Health Organisation, 1980:53.

4 Neimen RS, Barcos M, Berard C, Bonner H, Mann R, Rydall RE, et al. Granulocytic sarcoma: a clinicopathologic study of 61 biopsied cases. Cancer 1981;48:1426-37.

5 Meis JM, Butler JJ, Osborne BM, Manning JT. Granulocytic sarcoma in nonleukemic patients. Cancer 1986;58:2697709.

6 Zimmerman LE, Font RL. Ophthalmic manifestations of granulocytic sarcoma (myeloid sarcoma or chloroma). Am f Ophthalmol 1975;80:975-90.

7 Liu PI, Ishimaru T, McGregor DH, Akada H, Steer A. Autopsy study of granulocytic sarcoma (chloroma) in patients with myelogenous leukemia, Hiroshima-Nagasaki 1949-1969. Cancer 1973;31:948-55.

8 Dehner LP. Soft tissue sarcomas of childhood: the differential diagnosis of the small blue cell. Natl Cancer Inst Monogr 1981;56:43-59.

9 Hutchinson RE, Kurec AS, Davey FR. Granulocytic sarcoma. Clin Lab Med 1990;10:889-901.

10 Flavel DJ, Jones DB, Wright DH. Identification of tissue histiocytes on paraffin sections by a new monoclonal antibody. F Histochem Cytochem 1987;35:1217-26.

11 Hsu SM, Raine L, Fanger H. The use of avidin-biotinperoxidase complex (ABC) in the immunoperoxidase technique: a comparison between $\mathrm{ABC}$ and the unlabeled antibody (PAP) procedures. F Histochem Cytochem 1981;29: antibody

12 Cavdar AO, Babacan E, Gozdasoglu S, Kilcturgay K, Arcasoy A, Cin S, et al. High risk subgroup of acute myelomonocytic leukemia (AMML) with orbito-ocular granulocytic sarcoma (OOGS) in Turkish children: retrospective analysis of clinical, hematological, ultrastructural and therapeutic findings of thirty-three OOGS. Acta Haemat 1989;81:80-5.

13 Davies JNP, Owor R. Chloromatous tumours in African children in Uganda. BMF 1965;2:405-7.

14 Templeton AC. Orbital tumours in African children. $\mathrm{Br} \mathcal{F}$ Ophthalmol 1971;55:254-61.

15 Grossniklaus HE, Woino TH. Leukemic infiltrate appearing as periorbital cellulitis. Arch Ophthalmol 1990;108:484.

16 Ford JC, Yeatts RP, Hartz JW, Chauenet A. Granulocytic sarcoma of the eyelid as a presenting sign of leukemia. $\mathcal{F}$ Pediatr Ophthalmol Strabismus 1993;30:386-7.

17 Perry HD, Mallen FJ. Iris involvement in granulocytic sarcoma. Am f Ophthalmol 1979;87:530-2.

18 Brownstein S, Thelmo W, Olivier A. Granulocytic sarcoma of the orbit. Report of a case. Can $\mathcal{f}$ Ophthalmol 1975; 10:174-83.

19 Jordan DR, Noel LP, Carpentier BF. Chloroma. Arch Ophthalmol 1991;109:734.

20 Davies JL, Parke II DW, Font RL. Granulocytic sarcoma of the orbit. A clinicopathologic study. Ophthalmology 1985; 92:1758-62.

21 Mason TE, Demaree Jr RS, Margolis CI. Granulocytic sarcoma (chloroma), two years preceding myelogenous leukemia. Cancer 1973;31:423-32.

22 Whitcomb CC, Sternheim WL, Borowitz MJ, Davila E, Byrne GE. Case reports. T-cell lymphoma mimicking granulocytic sarcoma. Am f Clin Pathol 1985;84:760-2.

23 Brooks HW, Evans AE, Glass RM, Pang EM. Chloromas of the head and neck in childhood. The initial manifestation of the myeloid leukemia in three patients. Arch Otolaryngol 1974;100:306-8.

24 Pui MH, Fletcher BD, Langston JW. Granulocytic sarcoma in childhood leukemia: imaging features. Radiology 1994; 190:698-702.

25 Banna M, Aur R, Akkad S. Orbital granulocytic sarcoma. Am f NeuroRadiol 1991;12:255-8.

26 Davey FR, Olsen S, Kurec AS, Eastman-Abaya R, Gottlieb AJ, Mason D. The immunphenotyping of extramedullary myeloid cell tumours in paraffin-embedded tissue sections. Am f Surg Pathol 1988;12:699-707. 